\title{
Bridging content's quality between the participative web and the physical world
}

\author{
Anouar Abtoy ${ }^{1}$, Noura Aknin ${ }^{2}$, Boubker Sbihi ${ }^{1}$ \\ Ahmed El Moussaoui ${ }^{2}$ \& Kamal Eddine El Kadiri ${ }^{1}$. \\ ${ }^{1}$ LIROSA, ${ }^{2}$ LaSIT \\ Abdelmalek Essaadi University \\ Tétouan, Morroco
}

\begin{abstract}
- web 2.0 and its applications spread widely among internet users. Taking advantage of the easy, the friendly and the rich user interfaces. As consequence, the creation and the production of content become available to anyone. The ordinary user has step forward toward being a producer rather than remaining passive consumer. With this usage shifting, a new concern emerged: the quality of the User-Generated Content UGC or the User-Created Content UCC. Our team has developed a new concept of a Framework for managing the quality of the validated content in the participative web based on the evaluation of content's quality during its lifetime on the web. As continuation of our work, we will present a concept of extending the quality assessment of UGC or UCC into the real world by creating a bridge between digital content and its homologue in the physical world (e.g. printed version ...). We will combine existing technologies such as QR codes or RFID in order to perform the linking between the digital and the physical content. This approach well offers the possibility of following the quality of UGC or UCC; and eventually evaluates it; even in hard copies. The evaluation and the assessment of physical content originated from digital content generated through web 2.0 applications will be done in real-time. The proposed approach is implemented to our framework by integrating the features into the UML diagrams in the Blog case.
\end{abstract}

Keywords-Collaborative web; content management; digital content; physical content.

\section{INTRODUCTION}

Since its introduction Tim O'Reilly in 2004, web 2.0 has become an essential source of information for every single need in our era; this is mainly due the revolution of the concept of the web and its applications [1] [2]. The internet user has passed from being a simple consumer of content on the net to an active producer.

According to Tim O'Reilly [1], Web 2.0 brings new features to the Web thanks to the seven concepts which are:

- The Web is a services platform.

- The power of collective intelligence should be exploited efficiently.

- Data is the next Intel inside.

- End of the software release cycle.

- Lightweight programming models

- Software above the level of a single device.

- Rich user experiences.

The technological and the usage revolution of web 2.0 enabled various applications that took the web to a whole new level of surfing experience (Fig.1).

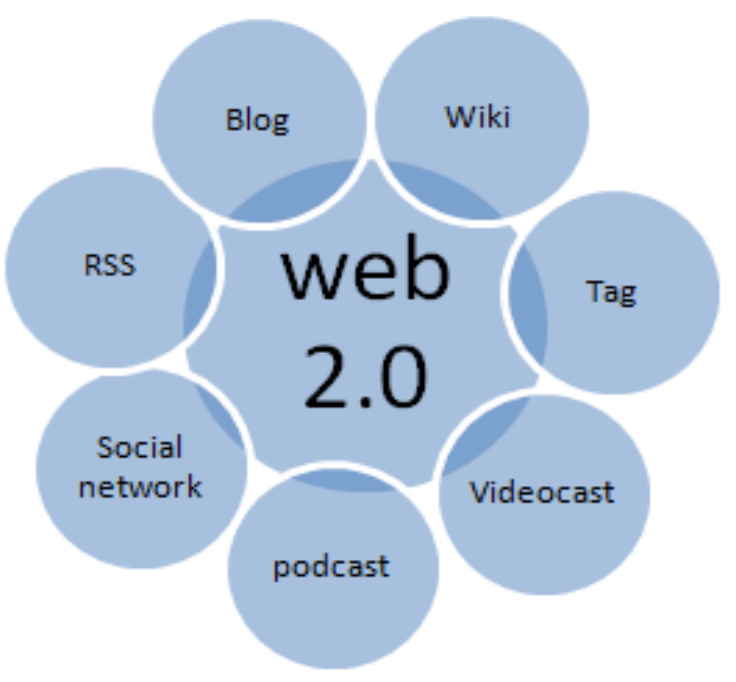

Figure 1. Web 2.0 applications

This change in the philosophy of content production and publication increases exponentially the mass of information available on the web. As a direct impact, new issues rise on the net. The lack of the quality and the relevance of both information and content are emerging as a major need for research in the area of the participative web [3].

At first we will introduce the new concept of content validation, and the validated Content Management Framework. Then, we will present our new vision content management for both digital and physical content in real-time.

\section{VALIDATED CONTENT MANAGEMENT FRAMEWORK}

The framework proposed by our team [4] is based on two major components: the categorization of information into types of quality, and the categorization of users into groups [3]:

- Categorization of information: unlike the earlier web, the information is subdivided into categories that represent a certain degree of information quality.

- Categorization of users: users are grouped into categories: users (eventually a producer), a validator and an expert. Each one of them has certain roles, responsibilities and privileges.

To ensure a certain quality of content, it is submitted to two validation processes: static and dynamic.

- Static validation: When a user produces content, it is submitted to an expert who assigns two validators whom 
will evaluate its relevance. Depending on their decision, the content will be published with an initial quality rating given by the combination of their note or it will be rejected (Fig.2).
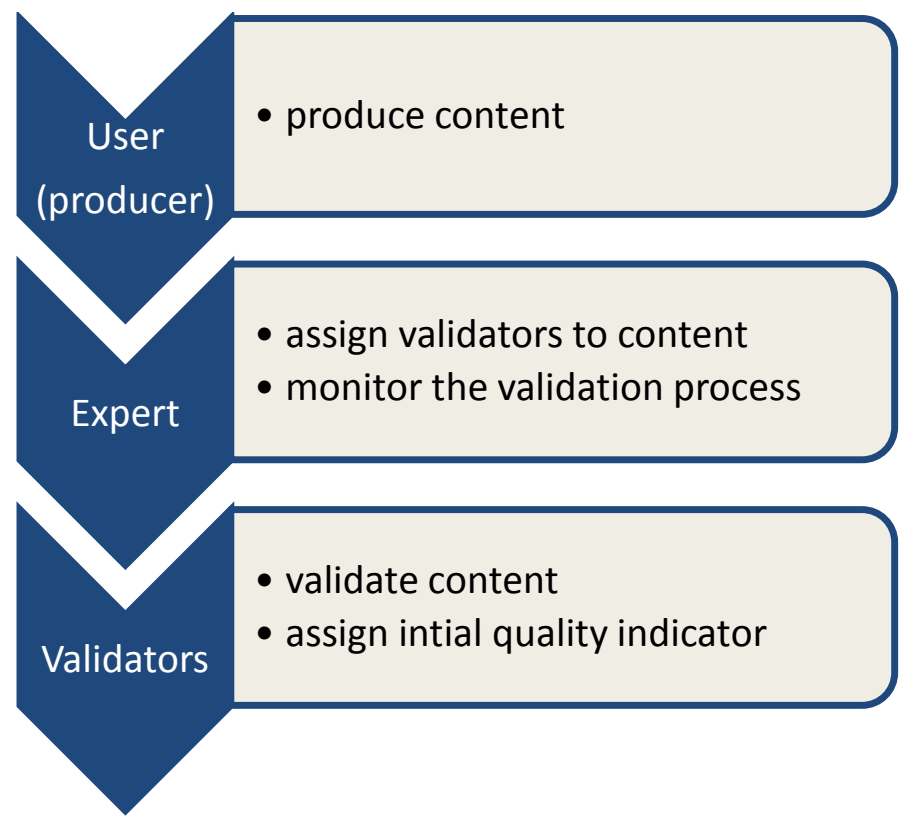

Figure 2. Static validation process [4]

- Dynamic validation: when content is validated and published through of a static validation on the net, the Internet community takes care of the ongoing validation of this content during its life on the web. The degradation of the quality of content till certain threshold causes the elimination or archiving of this content (Fig.3).

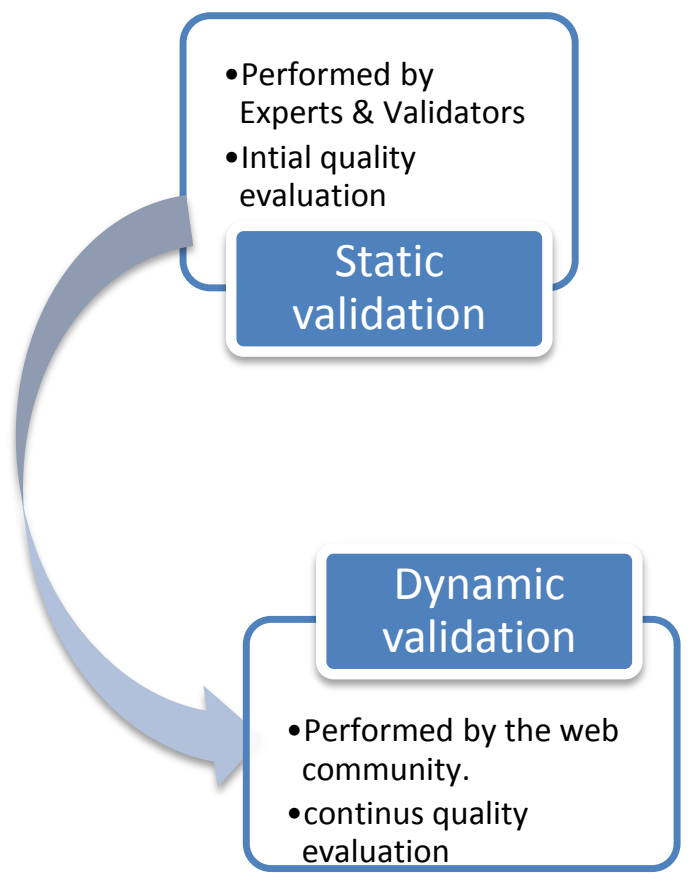

Figure 3. Static and dynamic validation complementary processes [4].

\section{BRIDIGING CONTENT QUALITY BETWEEN DIGITAL AND PYSICAL WORLD}

With easiness of accessibility of web 2.0 applications such as blogs, wikis and social networks, everyone can produce or generate a content which are called User-Generated Content $U G C$ or User-Created Content UCC. Based on our Framework, the content's quality can be monitored and evaluated form the moment of its creation to its elimination or archiving. This approach is valid as long as content remains in its digital form.

But usually, users tend to print digital content in order to keep printed version for offline reading. For multimedia content, users also tend to download it and put it on portable media player or burn it on CD, DVD, B-RD ....

The form shifting of content from digital to physical looses the connection between content and its quality indicator evaluated with our Framework.

The problem of linking the same content with different form was already discussed by researchers in other topics. An example this problem is the case of digital rights management for content distribution [5]. The difficulty was how to track and preserve digital rights of contents with continues changing form of those goods or assets (CD, DVD,Blu-ray, flash memory...) during distribution.

Our research is focused on the quality of User-Generated Content UGC or the User-Created Content UCC produced by ordinary users in the context of web 2.0 applications. The Framework that we presented [4] can give a way to evaluate UGC and UCC over the web. But once those content shift from digital to physical media, we need a new vision to assess and link the quality of these content with their original digital content.

We propose to add identification item to content in its physical form with certain tag that can be used to reach the original digital content on the net and its quality details. Our study focuses on two technologies: RFID and QR codes.

- RFID: Radio Frequency IDentification, it's a technology that uses tags in order to identify an object from certain distance unlike bar codes. It is used more and more on various application such as handling manufactured goods and materials [6]. This promising technology is tending to be combined with web services to offer presence-aware infrastructure for diverse application scenarios such as committee meeting scenario [7].

The RFID technology has been used in various researches to track digital content. It was proposed in the context of a Framework to mange digital rights and tracks it in its physical form such as digital mediums (Fig.4): CD, DVD or memory card [8]

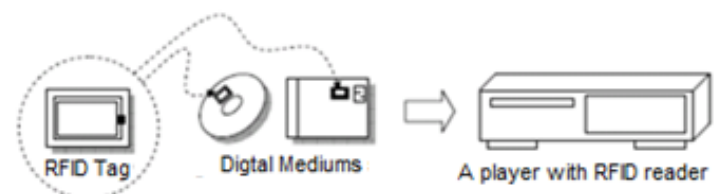

Figure 4. Tracking digital rights based on digital mediums and RFID Tags[8]

The RFID Tags can contain various information related to content, including URL that points to the content on the web.

- QR code: Quick Response code is 2 dimensional code or matrix barcode that was initially designed for automotive industry. QR codes offer more potential than the $1 \mathrm{D}$ barcodes. 


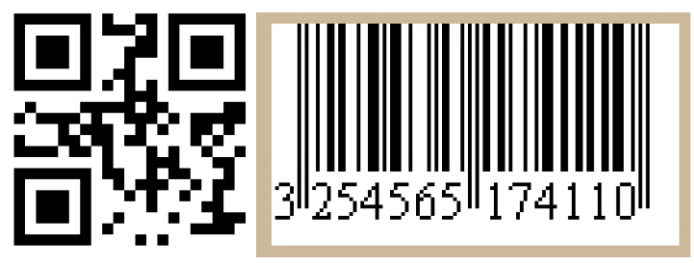

Figure 5. QR Code (2D) and EAN-13 (1D) barcodes encoding the same data (3254565174110)[6].

Some researches already used QR codes to link content with a web service by scanning the public QR code to obtain a URL in order to reach other information (Private part). The retrieved data are combined with the scanned to adapt contextual messages [9].

Linking print and digital content with QR codes is not a new concept [10], especially that any Smartphone become a QR code scanner. In our approach, we propose to link physical content (print or digital medium) via QR code with its correspondent digital content in the web. With this process, the user can know the current quality of the physical content that he possesses and eventually he can give his feedback and evaluate it.

Conceptually, the two proposed technologies are quite similar; both are intended to provide rapid and reliable item identification-and-tracking capabilities. The key difference between the two technologies is that $\mathrm{QR}$ code scans a printed label with imaging technology.

On the other hand, RFID scans or interrogates a tag using radio frequency signals. The table below summarizes the advantages and the inconveniences of both QR code and RFID:

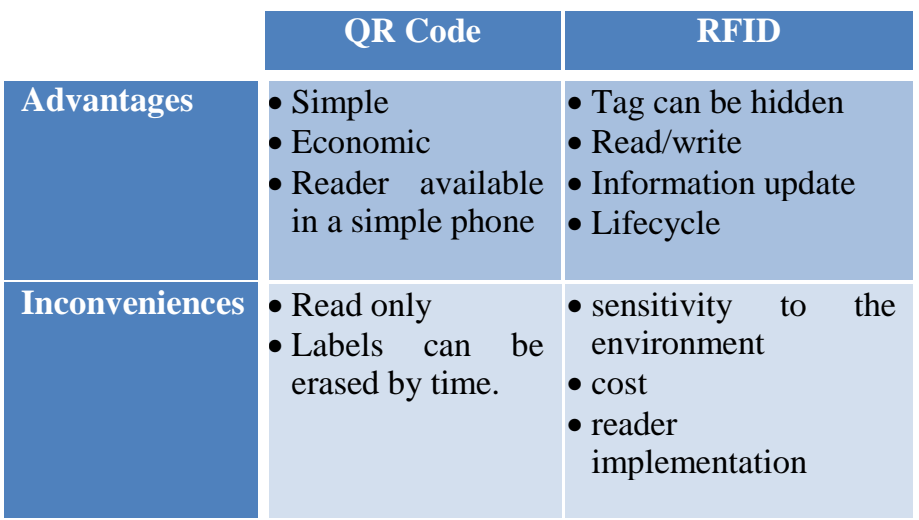

Table 1. The link between the quality of digital and physical content

Our approach relies on generating and integrating tags or labels (RFID or QR code) with physical version of the UGC or UCC. The evaluation of content's quality based on our approach it's a sustainable process. So when the content is printed or burned at certain moment $t_{n}$, the user can't know the state of this content's quality at $t_{n+1}$ based on its physical version.

Nowadays basic Smartphone offers the functionality to scan QR code labels, others can even be extended with RFID reader. Via the Tag in the content, the Smartphone can read it and then point to the unique digital version of the initial content on the web. Then, user can know the actual content's quality at $\mathrm{t}_{\mathrm{n}+1}$ and participate in its evaluation process.

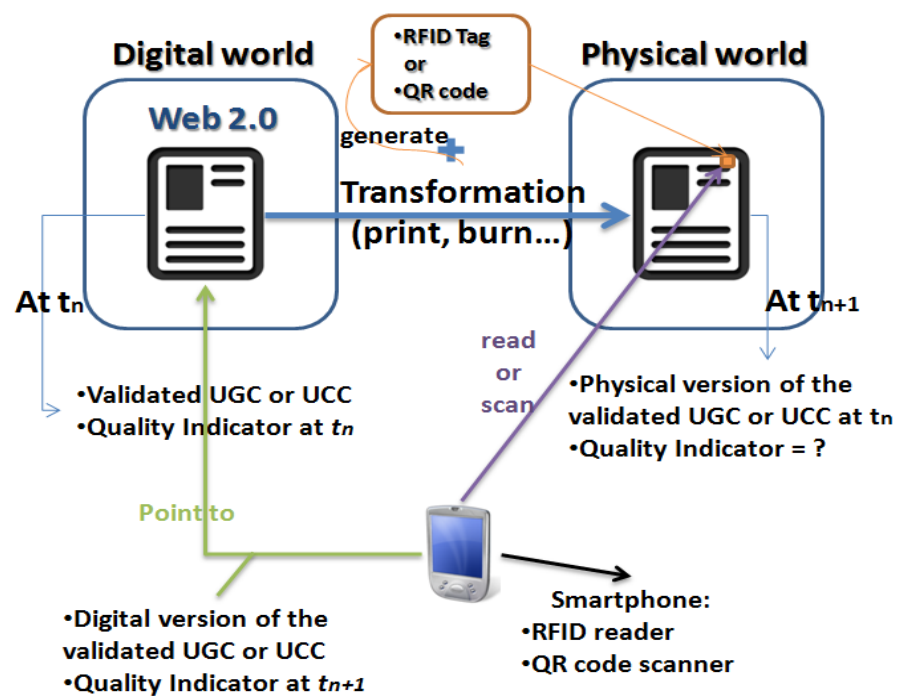

Figure 6. The link between the quality of digital and physical content [11]

\section{APPROACH IMPLANTATION: BLOG EXTENSTION CASE STUDY}

The framework of the validated content proposed by our team was implemented in a blog system to publish and mange validated blog posts $[4,11]$. The presented approach in this paper will bring new functionalities by extending the framework; therefore the modeling done in the previous work needs to be adjusted in order to reach the new requirements.

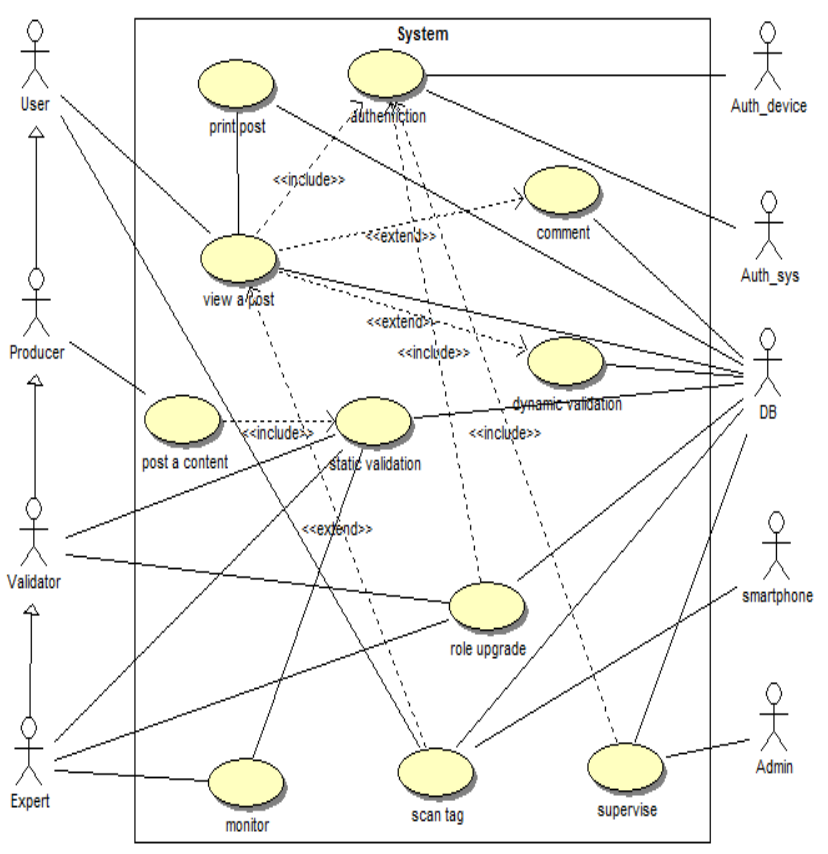

Figure 7. Use case diagram

The new use cases diagram includes new actor that was identified by analyzing the new requirements of the system. We modeled in this situation the Smartphone because in one hand, it becomes a popular device among users. On the other hand, the mobile technologies have known a breakthrough with technological development made on both levels: software (android, windows 7) and hardware (mobile processors architecture, high imaging capabilities) 
The uses case diagram previous use case diagram contains several use cases. Based on the new vision of validating both digital and physical content, the use case diagram (see figure below) will contain two new use cases: "scan tag" and "print post" use cases.

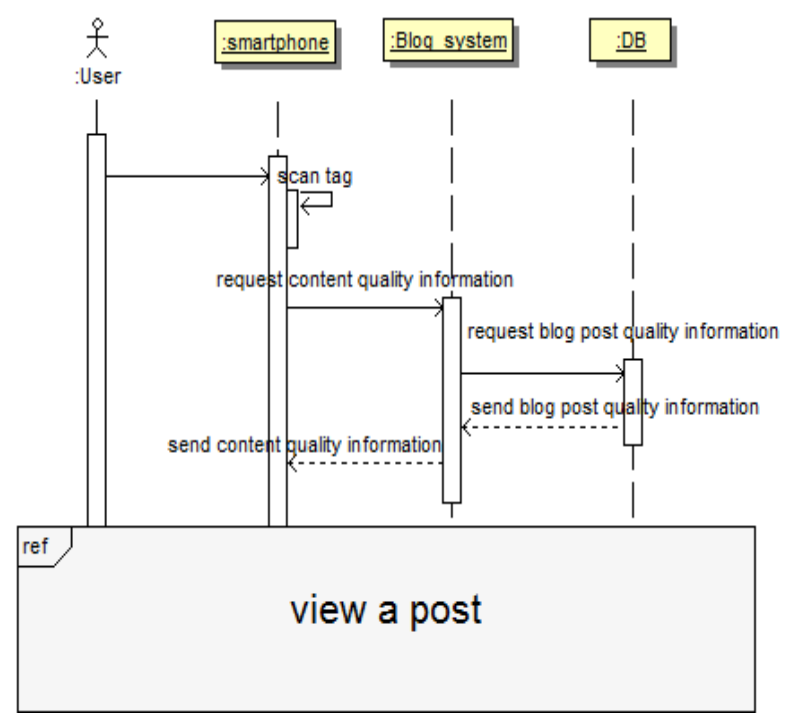

Figure 8. Scan tag sequence diagram

Each use case represent the new features integrated with the framework so that the system can deliver the quality information with physical version of the blog post, at the same time users can know the state of information quality including its validation state form the physical version.

In "scan tag" sequence diagram, the user uses his mobile device or Smartphone equipped by digital scanner for QR code to scan the tag of the physical version of blog post. Then, the Smartphone requests the blog post quality information from the system. As result, the system will send back this information to the user through his device. After accomplishing this phase, users have always the right to view the actual blog post content with its current quality information. Eventually, after authenticating, users can participate in the validation by dynamically validating the blog post.

Another possibility added to the system, is the preparation of the version that will be used to print the blog post or save it in a digital medium. The portable version is considered a prephysical version for the digital blog post. In our case, the suitable version is PDF (Portable Document Format) files.

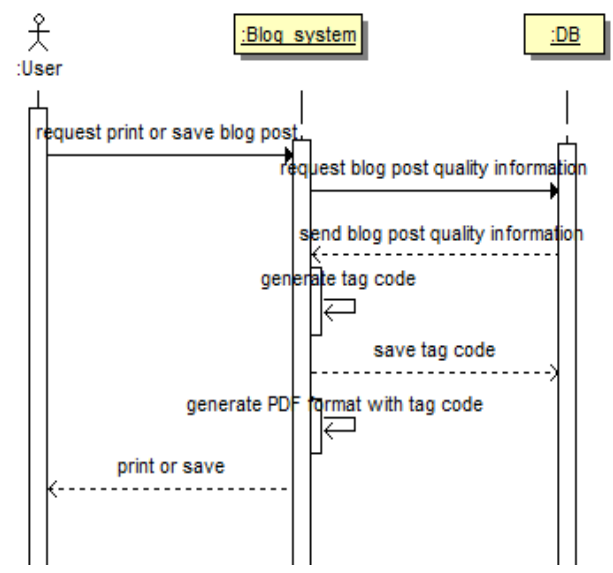

Figure 9. Print post sequence diagram
In the "print post" sequence diagram described above, the user requests from the blog system to save or print the current blog post. The Blog system retrieves the needed information from the database. The next step includes the generation of the tag by the system. In the case of QR codes technology, the system generates the necessary tag that points to the blog post and its quality information state. After saving the generated tag in the data base, the system precedes the generation of the blog post in its pre-physical version that includes the tag as a header. The finale phase is sending the final documents to the user so that he can print it or save in a digital medium for future purpose.

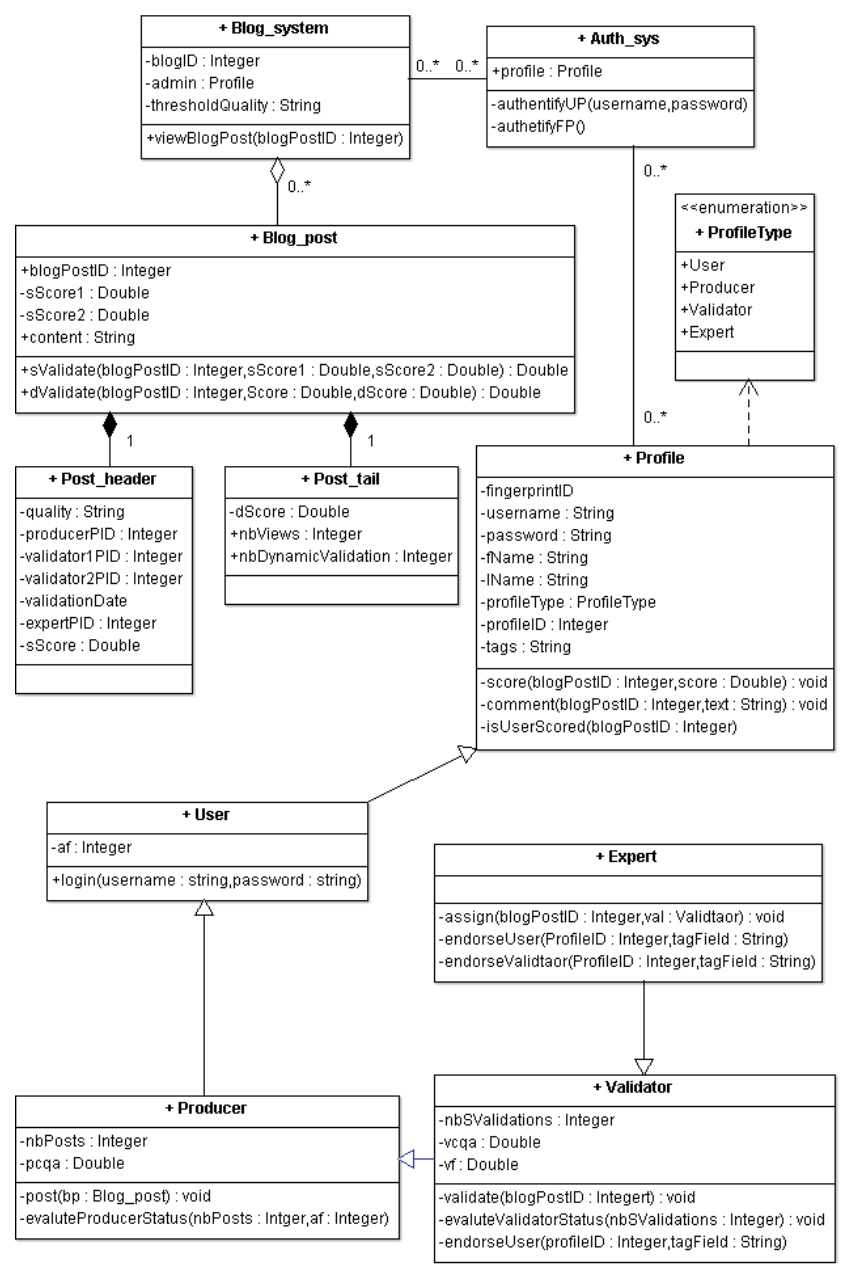

Figure 10. Class diagram of the enhenced blog system

The enhancements done in the system don't have implications on the class diagram molding (see figure 10). Mainly the generated QR code will point to the blog post based on its blogID attribute; therefore all the needed information will be retrieved based on this attribute form the system.

The enhanced blog system offers the possibility to create not only a validated blog posts with known quality, but also to create validated versions of the same content published within the system in their physical versions.

After integrating the approach with the validated content framework, it can be applied to other web 2.0 applications. The priority must be given to wiki systems because of their crucial role in the actual knowledge of users on the web.

The results obtained from our case study proved that bridging the concept of content's quality assessment between 
two different worlds (digital and physical) is possible. Also, the results in the case of Blogs encourage us to extend this approach in the case of other web 2.0 applications (Wikis, Social networks...)

\section{CONCLUSION}

The web 2.0 with its collaborative and participative feature encourages users to create and generate content easily. As a result, the web becomes a giant container for UGC and UCC. Our Framework for the management of the validated content offers the ability of to evaluate this information and content's quality over the web. But when it comes to the physical version of this content, it remains unable to assess and evaluates its quality. In this paper, we presented a new concept to extend our Framework to enclose even the physical content originated form participative web. We give an overview for the two possible technologies candidates for this process that are RFID and QR code. The implementation of the new approach into our framework is given in the case of blogs through UML diagrams.

The aim of this approach is bridging the physical and the digital world through simple technologies available to anyone. The potential is very promising and may lead to create a complete validated web that contains the UGC and UCC in their various formats and versions.

As perspective of our work, we propose to develop an approach of a system capable of tracking and classifying physical version of UGC/UCC. The purpose of this approach is to integrate the content into the new vision of internet of things.

\section{REFERENCE}

[1] T. O'Reilly, "what Is Web 2.0“, 2005, available at: http://oreilly.com/web2/archive/what-is-web-20.html.

[2] J.F Gervais, Web 2.0: les internautes au pouvoir, Edition, Dunod, 2007.

[3] B. Sbihi, and K.E. El Kadiri, "Web 2.2: Toward classified information on the Web", International Journal of Web Applications, Vol. 1, No. 2, Juin 2009, pp. 102- 109.

[4] A. Abtoy, N. Aknin, B. Sbihi, A. El Moussaoui and K.E. El Kadiri, "Towards a Framework for a validated content management on the collaborative Web: Blogs case", IJCSI International Journal of Computer Science Issues, Vol. 8, Issue 3, No. 2, May 2011, pp. 96-104.

[5] Q. Lui, R. Safavi-Naini, N.P. Sheppard, "Digital rights management for content distribution ",Proceeding ACSW Frontiers '03 Proceedings of the Australasian information security workshop conference on ACSW frontiers 2003 - Volume 21, 2003.

[6] R. Want, "An introduction to RFID technology", IEEE Pervasive Computing, Volume 5 Issue 1, Jan.-March 2006.

[7] C. Kerer, S. Dustdar, M. Jazayeri, D. Gomes, A. Szego, J. A. Burgos Caja, "Presence-Aware Infrastructure using Web services and RFID technologies", Proceedings of the 2nd European Workshop on Object Orientation and Web Services technologies, 2004 - Oslo, Norway.

[8] Suh, J.H. and S.C. Park, 2006. U-DRM: A Unified Framework of digital rights management based on RFID and Application of its usage data. International Journal of Computer and Network Security, 6 (8B): 151155.

[9] J. Rouillard, "Contextual QR Codes", Proceedings of the Third nternational Multi-Conference on Computing in the Global Information Technology. ICCGI 2008. July 27 - August 1, 2008 - Athens, Greece.

[10] D. A. MacRae, "Introducing QR Codes: Linking Print and Digital Content Via Smartphone", Neurosurgery, Volume 68, Issue 4, pp 854855, April 2011.

[11] A. Abtoy, Z. Itahriouan, N. Aknin, B. Sbihi, A. El Moussaoui and K.E. El Kadiri. "Towards quality assessment for both digital and physical Content." Proceedings of the third Mediterranean Symposium on Telecommunications, Fez - Morocco ,March 22,23 \& 24, 2012.

\section{AUTHORS PROFILE}

Anouar ABTOY received the Master degree in electronics and telecommunications in 2008 from Abdelmalek Essaadi University in Tetuan, Morocco. Currently, he is a PhD Student in Computer Science. He is also member of the Internet Society (ISOC) and student member of IEEE Computer Society. Ongoing research interests: Web 2.0, collaborative and collective intelligence, online identity, evaluation and assessment of information and content.

Noura AKNIN received the the PhD degree in Electrical Engineering in 1998 from Abdelmalek Essaadi University in Tetuan, Morocco. She is a Professor of Telecommunications and Computer Engineering in Abdelmalek Essaadi University since 2000. She is the Co-founder of the IEEE Morocco Section since November 2004 and she is the Women in Engineering Coordinator. She has been a member of the Organizing and the Scientific Committees of several symposia and conferences dealing with RF, Mobile Networks, Social Web and information technologies.

Boubker SBIHI received the PhD degree in computer science. Professor of computer science at the School of Information Science in Morocco. He is also the head of Information management department. He has published many articles on E-learning and Web 2.0. He is part of many boards of international journals and international conferences.

Ahmed EL MOUSSAOUI received the $\mathrm{PhD}$ degree in electronics at the University of BRADFORD in 1990. In 2007 he received the international master in E-learning in the Curt Bosh institute in Switzerland. He has been a member of the Organizing and the Scientific Committees of several symposia and conferences dealing with RF, Mobile Networks and information technologies. He has participated in several projects with France and Spain. Currently, his is the vice-president of Abdelmalek Essaadi University in Tetuan - Morocco.

Kamal Eddine EL KADIRI received the "Thèse de troisième cycles" degree in Data analysis at Paris VI University in 1984. He received "Thèse d'état" degree in Computer Science from Granada in 1994. . In 2007 he received the international master in E-learning in the Curt Bosh institute in Switzerland. He is a professor of Mathematics and Computer Science at Faculty of Sciences of Tetuan in Morocco. Currently, he is the director of the National School of Applied Sciences (ENSA) of Tetuan and also the director of LIROSA laboratory. He has published several articles on E-learning and Web 2.0. He is also part of many boards of international journals and international conferences. 